\title{
ERRATUM: “COSMIC-RAY ACCELERATION EFFICIENCY VERSUS TEMPERATURE EQUILIBRATION: THE CASE OF SNR 0509-67.5” (2010, ApJ, 719, L140)
}

\author{
E. A. Helder, D. KosenKo, And J. Vink \\ Astronomical Institute Utrecht, Utrecht University, P.O. Box 80000, NL-3508 TA Utrecht, The Netherlands; e.a.helder@astro-uu.nl \\ Received 2011 July 19; published 2011 August 4
}

\section{Online-only material: color figure}

In a recent email, K. Heng informed us that the curves he provided, which were used for Figure 2 of the published article, are incorrect. More specifically, Figure 5 of van Adelsberg et al. (2008) was the correct figure, in contrast to what was written in Helder et al. (2010).

Figure 2 here is the updated version of the corresponding published figure, utilizing the correct curves from van Adelsberg et al. (2008). This updated figure differs in two respects from the published figure. First, our measured FWHM and velocity for the NE

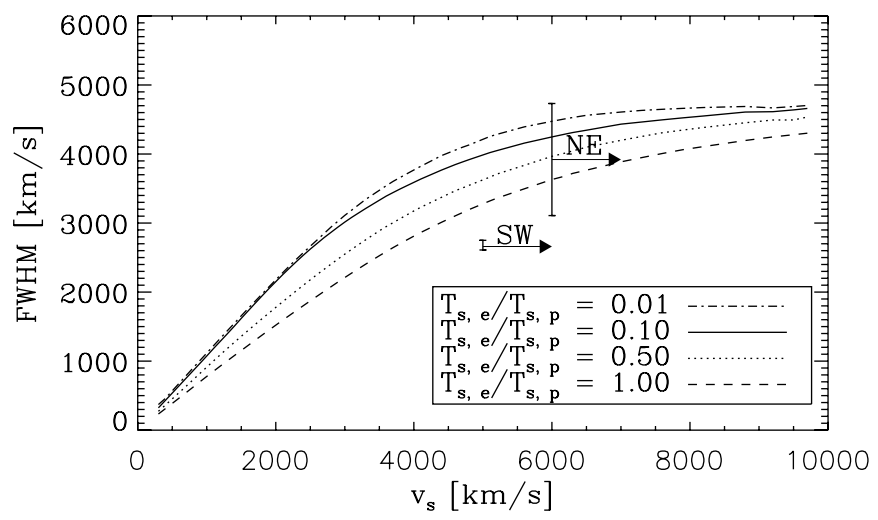

Figure 2. FWHM of the broad $\mathrm{H} \alpha$ component as a function of $v_{\mathrm{s}}$ for shocks without cosmic-ray acceleration, for different values for $T_{\mathrm{e}} / T_{\mathrm{p}}$. The overplotted data points are the estimated $v_{\mathrm{s}}$ and FWHM for both the SW and NE shocks.

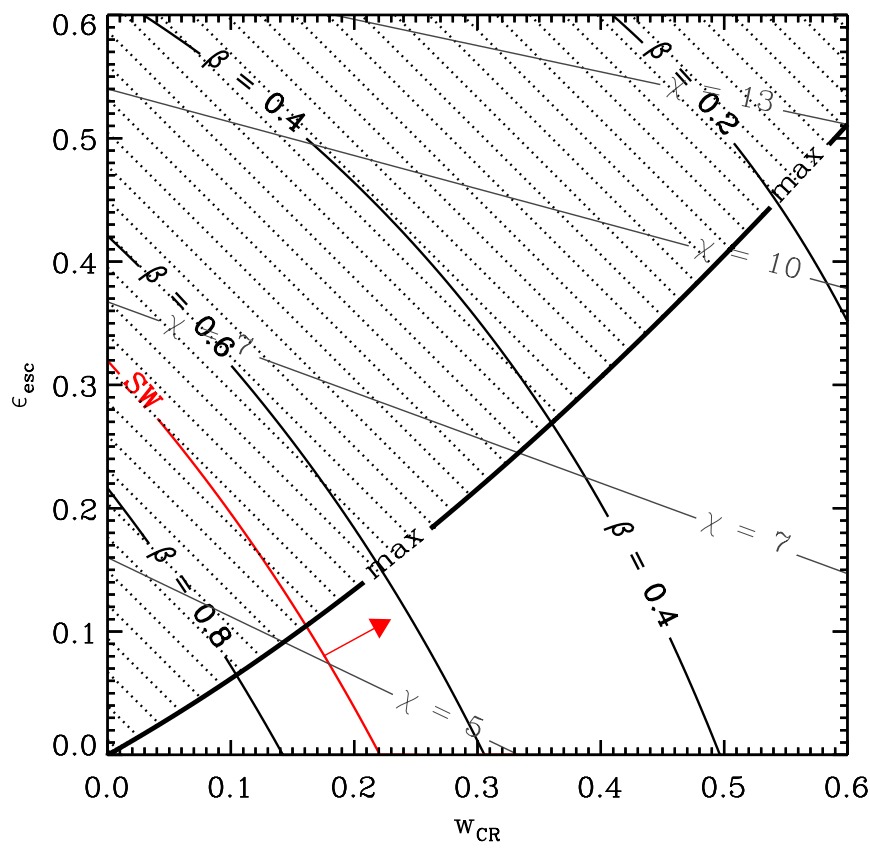

Figure 4. Values for $\beta\left(=\frac{k T_{\mathrm{s}, \mathrm{p}}}{3 / 16 \mu \mathrm{m}_{\mathrm{p}} v_{\mathrm{s}}^{2}}\right)$ in the ( $\left.w_{\mathrm{CR}}, \epsilon_{\mathrm{esc}}\right)$-frame, with $w_{\mathrm{CR}}$ being the fraction of the post-shock pressure contributed by cosmic rays, and $\epsilon_{\mathrm{esc}}$ the escape of cosmic-ray energy flux, normalized to the incoming energy flux. The $\chi$-lines indicate the compression ratio of the plasma behind the shock front. The hashed region requires a shock, more efficient than can be explained with current theoretical models. The red line indicates the lower limit for $\beta$ for the SW.

(A color version of this figure is available in the online journal.) 
shock now overlap all the curves for different $T_{\mathrm{s}, \mathrm{p}} / T_{\mathrm{s}, \mathrm{e}}$. This implies that from our measurement, we do not find evidence for a substantial cosmic-ray pressure behind the NE shock. Note however, that this does not necessarily mean that efficient cosmic-ray acceleration is excluded, as the measured shock velocity is a lower limit, and the large uncertainty on the measured FWHM includes the possibility that the FWHM is lower than predicted for a non-accelerating shock. Future proper motion studies of the shock will help to clarify this.

The measured FWHM and shock velocity for the SW shock still do not overlap any of the curves and hence, this implies that the SW shock is an efficient cosmic-ray accelerator. However, the lower limit for the $\beta$ value for the SW will be higher than originally reported: 0.7 instead of 0.58 , and the measured FWHM now leads to a post-shock proton temperature of $19.2 \pm 1.1 \mathrm{keV}$. Figure 4 here is the updated version of the corresponding published figure and shows that the cosmic-ray pressure $\left(w_{\mathrm{CR}}\right)$ behind the $\mathrm{SW}$ shock is $>15 \%$ of the total post-shock pressure.

\section{REFERENCES}

Helder, E. A., Kosenko, D., \& Vink, J. 2010, ApJ, 719, L140

van Adelsberg, M., Heng, K., McCray, R., \& Raymond, J. C. 2008, ApJ, 689, 1089 